\title{
Pulmonary Tuberculosis Caused By Immunosuppressive Treatment
}

\author{
Onal CO* and Kibaroglu E \\ Fourth Tuberculosis Dispensary, Ankara, Turkey
}

*Corresponding author: C Oztug Onal, Fourth Tuberculosis Dispensary, Ankara, Turkey, Tel: 00-90-312-3091224; E-mail: oztug26@yahoo.com

Received date: April 21, 2014, Accepted date: Jun 28, 2014, Published date: Jun 30, 2014

Copyright: (c) 2014 Onal CO, et al. This is an open-access article distributed under the terms of the Creative Commons Attribution License, which permits unrestricted use, distribution, and reproduction in any medium, provided the original author and source are credited.

\begin{abstract}
Immunosuppressive treatment is used in many clinical conditions. These drugs help to suppress immune system which functions excessively or optimum level, while creating some risks as well. The most important of these risks is the disappearance of defense against infectious agents and emergence of undue infections. Therefore, during immunosuppressive treatment, prophylactic anti-infective agents may be required. In this case presentation a male patient at the age of 47 who has been on immunosuppressive treatment for twenty years with the diagnosis of ankylosing spondylitis and diagnosed tuberculosis is presented.
\end{abstract}

Keywords: Immunosuppressive treatment; Tuberculosis; Opportunistic infections

\section{Introduction}

Tuberculosis (TB) remains an important health problem in developing countries. Clinical manifestations depend on the location of the TB, which can involve any organ of the body [1]. The World Health Organization estimated that 8.8 million new cases of tuberculosis (TB) and 1.45 million deaths due to TB occurred worldwide in 2009, and almost 160 people die of TB each hour [2]. Immunosuppressive treatment is used in many clinical conditions. Among these are autoimmune diseases such as ankylosing spondylitis, rheumatoid arthritis, sarcoidosis, Behçet's disease, multiple sclerosis, organ transplantation and some inflammatory diseases, which are not autoimmune. Agents used for immunosuppressive purposes have been classified into five groups, i.e. glucorticoids, cytostatics, antibodies, drugs acting on immunophilins and other drugs such as interferon. These drugs help to suppress immune system which functions excessively or optimum level, while creating some risks as well. The most important of these risks is the disappearance of defense against infectious agents and emergence of undue infections. Therefore, during immunosuppressive treatment, prophylactic anti-infective agents may be required. Numerous prospective studies and case reports have underlined the tendency of patients who used to immunosuppressive therapy to develop severe infections because of the opportunistic and common microbial pathogens $[3,4]$.

This multimodal approach to immunosuppressive therapy significantly changes the overall immunity of patients, increasing their risk for opportunistic infections.

Therapeutic blockade of tumour necrosis factor alpha (TNFa) has been developed as an effective treatment in immune-mediated inflammatory diseases (IMID) such as Crohn's disease, ankylosing spondylitis, rheumatoid arthritis and psoriatic arthritis. However, $\mathrm{TNFa}$ is a key cytokine in protective host defence against Mycobacterium tuberculosis (M. tuberculosis). It plays an important role together with TNF-dependent chemokines [5], in the development and maintenance of the granuloma which compartmentalises tubercle bacilli during infection [6,7]. In patients latently infected with $\mathrm{M}$. tuberculosis, inhibition of TNFa and TNFregulated chemokine networks constitutes the presumed biological basis for the 4 to 5 fold higher incidence of tuberculosis observed after initiation of anti-TNF therapy with infliximab $[8,9]$. Therefore, it has been demonstrated that, exclusion of active TB and treatment of latent tuberculosis infection (LTBI) are clinical imperatives prior to initiating anti-TNF therapy and active surveillance for a history of untreated or partially treated TB or LTBI and effective in reducing the number of incident TB cases $[9,10]$.

\section{Case}

The male patient at the age of 47 had a history of ankylosing spondylitis since 1983. He has been on immunosuppressive treatment for twenty years. Since analgesic anti-inflammatory drugs and glucocorticids did not resolve the symptoms, it was decided on September 2012 that TNFa antagonist monoclonal antibody should be initiated and various anti-TNF treatments (adalimumab, etanercept and infliximab) had been prescribed.

Patient was evaluated for tuberculosis (TB) by TB Mendel-Mantoux skin test before immune suppression treatment and when Tuberculin Skin Test (TST) result was found to be $6 \mathrm{~mm}$, it was recommended that patient should use prophylactic Isoniazid $300 \mathrm{mg} /$ day for 9 months. At first month control visit, no side effects of Isoniazid were observed. After one month, patients discontinued Isoniazid as it induced his appetite too much and did not share this information with his physician. In 2013 March, he referred again with the complaints of cough, loss of weight and fatique, which continued for a month without resolution. On chest X-ray which yielded mild diffuse interstitial infiltrates and chest computed tomography showed significant bilateral lung tissue opacities and pericardial effusion and sputum smear examination, patient was diagnosed to have smear positive pulmonary TB. Following the collection of multiple additional samples for microbiological investigations, a calculated antituberculosis quadruple treatment was immediately initiated with rifampicin, isoniazid, ethambutol and pyrazinamide. Of those, citrate blood sample, TB PCR from sputum and staining's for acid-fast bacilli from bronchoalveolar lavage as well as lung biopsy were negative. Drug susceptibility testing yielded that the patient's microbic agent was sensitive to all TB drugs. After four drug treatment for two 
Page 2 of 3

months, it was decided to maintain Isoniazid and rifampicin treatment until the completion of immunosuppressive treatment.

\section{Discussion}

Patients presented with the classic but non-specific symptoms such as cough, fever, and weight loss should be considered at risk for tuberculosis, especially if they come from a high-prevalence country and are immunosuppressed with a low sensitivity for the detection of parenchymal lung abnormalities [11]. Accordingly, previous chest Xrays had been normal in our patient at admission to the primary care hospital.

However, the subsequent chest X-rays upon admission to our hospital were suggestive for miliary $\mathrm{TB}$ as diffuse infiltrates evolved over the course of the disease [12]. Opportunistic infections are resulted from organisms that take advantage of a weakened immune system and lead to disease when they ordinarily would cause mild illness or no disease in the immunocompetent host. Opportunistic infections are associated with significant mortality and morbidity in individuals with a compromised immune system [13]. The risk factors for opportunistic infections include congenital immunodeficiency, HIV infection, chronic diseases such as diabetes mellitus and emphysema, malnutrition, older age and use of immunosuppressive medications such as immunomodulators (methotrexate, thiopurines) corticosteroids and anti-TNFa therapy [14,15]. Furthermore, the use of thiopurines (azathioprine, 6-mercaptopurine (6-MP)) corticosteroids, and anti-TNFa agents were associated with an increased risk. Combination of these agents increased the risk manifold [4]. The reactivation risk of latent $\mathrm{TB}$ is significantly associated with receiving TNFa inhibitors, with a 5-fold higher risk of reactivation in the first 52 weeks after initiation of therapy in the patients receiving TNFa inhibitors [10,16,17]. This may be the reason for TNFa to be essential for granuloma formation that is responsible for sequestration of mycobacteria [16]. Thus, inhibition of TNFa may cause reactivation of latent infection. A recent retrospective study reported a reactivation rate of latent TB infection by $19 \%$, despite chemoprophylaxis in patients with IBD on anti-TNFa therapy, thus warranting routine TB surveillance in these patients after initiating anti-TNFa drugs, even after completion of chemoprophylaxis [18]. There are no any other study reporting optimum timing for initiating anti-TNFa therapy after chemoprophylaxis for latent TB, although it has been generally recommended to wait for at least a month or 2 after initiation of chemoprophylaxis $[16,19]$.The respective sensitivities of the applied tests must be critically taken into account to rule out the differential diagnosis of gastrointestinal and miliary TB, especially in immunosuppressed patients [12].

In a study published in 2013, the age range of $\mathrm{Tb}$ cases occurring in patients using TNFa inhibitor was found to be between 32-67. In this study carried out by Chung et al, of 7 patients, only One patient received isoniazid chemoprophlaxy. Two patients were diagnosed with TB and as in our cases, they used HREZ treatment protocol. Patients indicated for Isoniazid chemoprophlaxy should be adequately and constantly trained for compliance to latent TB treatment. In a more resent study, Alawneh et al also demonstrated that in patients using TNFa inhibitor, TB developed. 2 of these patients were diagnosed with TB and with disseminated TB. Anti-TNFa treatment can activate all forms of TB [20,21].

\section{Conclusion}

Before initiating immunosuppressive treatment, strategy should be developed against common infectious diseases in the community. Especially before the use of agents impairing cellular immunity such as TNFa antagonists, subject should be evaluated for tuberculosis and chemoprophylaxis administered if necessary. TST may yield false negative results due to previously used immunosuppressive drugs like glucocorticoids. That's why the decision of chemoprophylaxis is both difficult and important. Patients considered for immune suppressive treatment should be protected against opportunistic infections. Informing patients adequately on chemoprophylaxis will prevent adverse outcomes. Treatment should not be spared because of negative tests in a high risk scenario for TB. Finally, intestinal TB should always be kept in mind as a differential diagnosis in unusual clinical courses of suspected inflammatory bowel disease under anti-TNF therapy [12].

\section{References}

1. Zumla A, Raviglione M, Hafner R, von Reyn CF (2013) Tuberculosis. N Engl J Med 368: 745-755.

2. Millet JP, Moreno A, Fina L, del Baño L, Orcau A, et al. (2013) Factors that influence current tuberculosis epidemiology. Eur Spine J 22 Suppl 4: 539-548.

3. Azie N, Neofytos D, Pfaller M, Meier-Kriesche HU, Quan SP, et al. (2012) The PATH (Prospective Antifungal Therapy) Alliance registry and invasive fungal infections: update 2012. Diagn Microbiol Infect Dis 73: 293-300.

4. Lichtenstein GR, Feagan BG, Cohen RD, Salzberg BA, Diamond RH, et al. (2012) Serious infection and mortality in patients with Crohn's disease: more than 5 years of follow-up in the TREATâ,, $₫$ registry. Am J Gastroenterol 107: 1409-1422.

5. Newton SM, Mackie SL, Martineau AR, Wilkinson KA, Kampmann B, et al. (2008) Reduction of chemokine secretion in response to mycobacteria in infliximab-treated patients. Clin Vaccine Immunol 15: 506-512.

6. Chakravarty SD, Zhu G, Tsai MC, Mohan VP, Marino S, et al. (2008) Tumor necrosis factor blockade in chronic murine tuberculosis enhances granulomatous inflammation and disorganizes granulomas in the lungs. Infect Immun 76: 916-926.

7. Algood HM, Lin PL, Flynn JL (2005) Tumor necrosis factor and chemokine interactions in the formation and maintenance of granulomas in tuberculosis. Clin Infect Dis 41 Suppl 3: S189-193.

8. Keane J, Gershon S, Wise RP, Mirabile-Levens E, Kasznica J, et al. (2001) Tuberculosis associated with infliximab, a tumor necrosis factor alphaneutralizing agent. N Engl J Med 345: 1098-1104.

9. Wolfe F, Michaud K, Anderson J, Urbansky K (2004) Tuberculosis infection in patients with rheumatoid arthritis and the effect of infliximab therapy. Arthritis Rheum 50: 372-379.

10. Gomez-Reino JJ, Carmona L, Valverde VR, Mola EM, Montero MD, et al. (2003) Treatment of rheumatoid arthritis with tumor necrosis factor inhibitors may predispose to significant increase in tuberculosis risk: a multicenter active-surveillance report. Arthritis Rheum 48: 2122-2127.

11. Geng E, Kreiswirth B, Burzynski J, Schluger NW (2005) Clinical and radiographic correlates of primary and reactivation tuberculosis: a molecular epidemiology study. JAMA 293: 2740-2745.

12. Koschny R, Junghanss T, Mischnik A, Karner M, Kreuter M, et al. (2013) Development of miliary tuberculosis under infliximab in a patient with spondyloarthritis and suspected Crohn's disease. Z Gastroenterol 51: 1177-1183.

13. Toruner M, Loftus EV Jr, Harmsen WS, Zinsmeister AR, Orenstein R, et al. (2008) Risk factors for opportunistic infections in patients with inflammatory bowel disease. Gastroenterology 134: 929-936.

14. Rahier JF, Ben-Horin S, Chowers Y, Conlon C, De Munter P, et al. (2009) European evidence-based consensus on the prevention, diagnosis and 
Citation: Onal CO, Kibaroglu E (2014) Pulmonary Tuberculosis Caused By Immunosuppressive Treatment. J Clin Case Rep 4: 383. doi: 10.4172/2165-7920.1000383

Page 3 of 3

management of opportunistic infections in inflammatory bowel disease. J Crohns Colitis 3: 47-91.

15. Doran MF, Crowson CS, Pond GR, O'Fallon WM, Gabriel SE (2002) Predictors of infection in rheumatoid arthritis. Arthritis Rheum 46: 2294-2300.

16. Afif W, Loftus EV Jr (2010) Safety profile of IBD therapeutics: infectious risks. Med Clin North Am 94: 115-133.

17. Qumseya BJ, Ananthakrishnan AN, Skaros S, Bonner M, Issa M, et al. (2011) QuantiFERON TB gold testing for tuberculosis screening in an inflammatory bowel disease cohort in the United States. Inflamm Bowel Dis $17: 77-83$

18. Sichletidis L, Settas L, Spyratos D, Chloros D, Patakas D (2006) Tuberculosis in patients receiving anti-TNF agents despite chemoprophylaxis. Int J Tuberc Lung Dis 10: 1127-1132.
19. Theis VS, Rhodes JM (2008) Review article: minimizing tuberculosis during anti-tumour necrosis factor-alpha treatment of inflammatory bowel disease. Aliment Pharmacol Ther 27: 19-30.

20. Keun Bum Chung, Eun Young Lee, Jong Pil Im, Sung Koo Han, Jae-Joon Yim (2013) Clinical characteristics and treatment responses of patients who developed tuberculosis following use of a tumor necrosis factor-a inhibitor. Korean J Intern Med 28: 174-179.

21. Alawneh KM, Ayesh MH, Khassawneh BY, Saadeh SS, Smadi M, et al. (2014) Anti-TNF therapy in Jordan: A focus on severe infections and tuberculosis. Biologics: Targets and Therapy 8: 193-198. 